\title{
LE CÔTÉ LUMINEUX DE LA FORCE DU SOCIAL. EN HOMMAGE SOCIOPHILIQUE À LA COSMOPHILIE D’HENRI RAYNAL
}

Philippe Chanial

\author{
La Découverte | « Revue du MAUSS »
}

2018/1 n $51 \mid$ pages 271 à 286

ISSN $1247-4819$

ISBN 9782348036064

Article disponible en ligne à l'adresse :

https://www.cairn.info/revue-du-mauss-2018-1-page-271.htm

\section{Pour citer cet article :}

Philippe Chanial, « Le côté lumineux de la force du social. En hommage sociophilique à la cosmophilie d'Henri Raynal », Revue du MAUSS 2018/1 (n 51), p. 271-286.

DOI $10.3917 / \mathrm{rdm} .051 .0271$

Distribution électronique Cairn.info pour La Découverte.

(C) La Découverte. Tous droits réservés pour tous pays.

La reproduction ou représentation de cet article, notamment par photocopie, n'est autorisée que dans les limites des conditions générales d'utilisation du site ou, le cas échéant, des conditions générales de la licence souscrite par votre établissement. Toute autre reproduction ou représentation, en tout ou partie, sous quelque forme et de quelque manière que ce soit, est interdite sauf accord préalable et écrit de l'éditeur, en dehors des cas prévus par la législation en vigueur en France. Il est précisé que son stockage dans une base de données est également interdit. 


\title{
Le côté lumineux de la force du social \\ En hommage sociophilique à la cosmophilie d'Henri Raynal $^{1}$
}

\author{
Philippe Chanial
}

\begin{abstract}
« J'aime ce qui est lié. Fût-ce de façon quasi invisible, immatérielle, toute aérienne, comme les figures auxquelles se prête la grâce, comme les éléments dont est faite et qu'entraîne la mélodie. Peutêtre que l'Être entier converge lentement vers une cérémonie. »
\end{abstract}

Henri Raynal, L'Eil magique.

Et si, à prétendre, inlassablement, lever le voile de notre ignorance de la vérité du monde social, les lumières des sciences sociales, du moins dans leurs paradigmes contemporains dominants, nous plongeaient, paradoxalement, dans l'ombre ? Et si, à tant chercher à dessiller et désenvoûter notre regard, elles nous aveuglaient et, d'un mauvais œil, nous frappaient de cécité ? C'est à partir de telles questions que je voudrais ici, en hommage à l'œuvre du poète Henri Raynal, interroger la lucidité de la « critique critique » et cheminer en sa compagnie vers ce que j'ai nommé la « délicate essence du social » [Chanial, 2011].

Je n'ai jamais lu Henri Raynal en sociologue, mais bien davantage comme une échappée belle, loin de ma discipline. Or, paradoxalement, cette échappée belle n'a cessé de me reconduire à elle.

1. Cet article reprend pour l'essentiel notre contribution à l'ouvrage d'hommages rendus à l'œuvre d'Henri Raynal [Boblet, Canonne, 2018]. 
Comme chacun sait, une échappée belle désigne tout à la fois une escapade dans un endroit agréable, une fuite loin d'une morosité pesante, voire une brèche lumineuse, et le fait d'éviter, de justesse, un danger, un péril. En ce double sens, grâce à la lecture d'Henri Raynal, je l'ai doublement échappé(e) belle. Sa Cosmophilie ${ }^{2}$ m'a aidé, d'une part, à résister à l'attraction qu'exerce sur nombre de sociologues le côté obscur de la force du social ${ }^{3}$, à m'immuniser contre cette maladie sociologique assez répandue, que je propose de désigner sous le terme de sociophobie. D'autre part, son invitation à prendre le «parti du lumineux contre celui de l'assombrissement» [Raynal, 2010, p. 53] m'a renforcé dans l'idée qu'il n'était en rien naïf de porter son regard sur la face lumineuse de cette force du social, et ainsi frayer la voie vers une sociologie résolument sociophilique.

Ce cheminement est à l'évidence semé d'embûches puisqu'il suppose non seulement de justifier ce diagnostic sévère d'une sociophobie sociologique, mais surtout d'ouvrir la cosmophilie raynalienne à une sociophilie qui lui semble presque étrangère ${ }^{4}$. Tentons néanmoins l'aventure.

\section{Lorsque la lucidité s'aveugle}

Le titre d'un récent ouvrage de Luc Boltanski [2008], Rendre la réalité inacceptable, où il raconte l'esprit dans lequel il menait son travail sociologique dans la bande à Bourdieu, résume bien ce diagnostic clinique de sociophobie. Comme si les sciences sociales supposaient, à titre de geste fondateur, de rompre avec toute « affinité », toute " confraternité » (Henri Raynal) avec le monde ; comme si, par conséquent, l'énigme même de l'ordre social - le fait qu'il y ait, parmi ou entre les hommes, quelque chose plutôt que rien (le chaos, l'arbitraire de la violence, la guerre de

2. Tel est le titre de son dernier recueil.

3. Sa réduction à la violence, à la contrainte, au pouvoir, à la domination, à l'aliénation, pour le dire en un mot.

4. Rares sont en effet les pages dans lesquelles l'auteur évoque ou célèbre un merveilleux objectif présent dans le monde des relations interhumaines et dont les sciences sociales auraient vocation à témoigner. Au point de se demander si le socius (à la différence de la physis) a sa place dans le cosmos raynalien. 
tous contre tous, etc.) ou, pour le dire autrement, le fait que ceux-ci parviennent à donner forme à leur coexistence - n'avait rien de miraculeux et pouvait, au contraire, se résoudre dans le dévoilement de l'arbitraire social ; comme si d'obscures relations de pouvoir faisaient seules tenir ce monde et imposaient ces formes de la coexistence interhumaine. Bref, le monde social ne serait autre que le royaume de l'illusio, pour emprunter ce terme à Pierre Bourdieu, et le sociologue le chef de ce qu'Henri Raynal nomme la « coalition des désillusionneurs» [Raynal, 2012, p. 20].

Dans un texte bien nommé, «Louée soit l'Illusion » [ibid.], l'auteur rappelle d'ailleurs combien « dissiper les illusions» constitue le «sport intellectuel favori » de notre époque au point que celle-ci «échoue à rendre compte d'un objet qu'elle détruit sous elle ». Sous certains de ses aspects, la sociologie contemporaine est symptomatique d'une telle époque et, par là, tend à détruire son objet même : le mystère de l'ordre social. En ce sens, en sociologie aussi, souvent, « la lucidité s'aveugle» [ibid.].

Comment, alors, surmonter cette cécité ? S'émerveiller, c'est tout simplement bien voir, suggère Henri Raynal dans L'Accord [2010, p. 53]. Mais que peut bien signifier « bien voir » pour les sciences sociales ? Et de quelle merveille pourraient-elles témoigner? Dans un entretien publié en 2002 [Raynal, 2002, p. 9], l'auteur suggère que témoigner consiste à « rendre justice à l'Inventivité énigmatique » à l'œuvre dans la Nature, et invite à « considérer le monde comme le théâtre de l'actualisation des possibles ». Si l'on suit cette suggestion et cette invitation, faire droit au côté lumineux de la force du social suppose de rendre justice à ce qui est ${ }^{5}$, à cette inventivité énigmatique qui se manifeste également dans la texture, dans la chair même du social ; de témoigner d'un « merveilleux objectif » [Raynal, 2016,p. 32] à l'œuvre dans le monde social, d'un «trésor des possibles » déposés au cœur des relations interhumaines et dans le mouvement d'ensemble qui s'y dessine. Tentons donc

5. «Reconnaitre le plus grand droit de l'objet, son droit imprescriptible, opposable à tout poème... Aucun poème n'étant jamais sans appel a minima de la part de l'objet du poème ni sans plainte en contrefaçon », écrivait Francis Ponge, en introduction de La Rage de l'expression (1952). Étrangement, ce poète, si proche de l'inspiration d'Henri Raynal, est convoqué par Pierre Bourdieu pour justifier son « parti pris » du réel et son ambition de dévoiler la vérité de la souffrance sociale et de la «misère du monde [1993, p. 1400]. 
de cheminer de l'ombre à la lumière en décrivant tout d'abord les symptômes de cette maladie sociologique appelée sociophobie, pour, ensuite, éclairer certains aspects, indissociables, de la délicate et lumineuse essence du social : sa dimension intrinsèquement morale, épiphanique et esthétique.

\section{La sociophobie et la fleur vivante}

Contre toute accusation d'irénisme, relisons cet extrait d'un dialogue du roman L'Accord:

N'allez pas croire que nous ignorions le détestable, le déplorable, le haïssable - la souffrance, la violence, le conflit, l'iniquité, la vilenie. Nous ne nous dissimulons pas le négatif : nous contestons la place abusive qui lui est consentie trop souvent. En opposition à l'accaparement des esprits par le déplorable, au détriment du remarquable, de l'admirable, du louable, nous avons choisi de nous consacrer à recueillir la qualité qu'une générosité, anonyme ou humaine, dispense, à la présenter de façon à ce qu'elle puisse se montrer en sa légitime évidence [Raynal, 2010 ${ }^{6}$.

Ce plaidoyer pour le parti du lumineux permet, en premier lieu, de refuser que cette «pensée du déplorable » s'attribue le monopole de la réalité. Plus encore, il invite à pointer combien la fascination de nombre de sociologues pour le côté obscur de la force du social les rend en partie incapables de rendre justice à ce qui est, et fait d'eux, paradoxalement, des « déréalistes » [Raynal, 2012, p. 20]. En effet, s'il est rarement question, en sociologie, de rendre justice à ce qui est, c'est parce que, manifestement, ce qui est ne vaut pas un rond. Dès lors, selon Emmanuel Renault, la tâche du sociologue consiste avant tout à faire apparaître « ce monde dans ce qu'il a de tout bonnement insupportable » [Renault, 2004,p. 406]. Dans cette rhétorique de la dénonciation, il s'agit de montrer que le monde n'est pas ce qu'il devrait être. Il est immonde, répugnant, à vomir ou hypocrite, tant il ne fait pas ce qu'il dit, ne dit pas ce qu'il fait. Il nous ment.

Pire encore, il nous aliène. D'où cette seconde prétention de la sociologie critique : «Décrire pour détacher les subjectivités de ce

6. Voir également Raynal [2016, Livre I, chap. I : « Cosmophlie vs décivilisation»]. 
monde qui est notre ennemi », pour mieux saper les " modalités d'identification au monde » [ibid.]. Il s'agit alors de rendre raison de notre aveuglement : les sujets sont comme frappés de cécité (mystifiés) face à ce qui est (la réalité leur échappe au point d'être soumis à ce qui est) ; de « rendre raison de notre culpabilité inconsciente » : les sujets sont, directement ou indirectement, responsables de ce qui est - ils sont empêtrés, englués, de façon complice, dans ce qui est, au point d'être étrangers à ce qu'ils devraient être ${ }^{7}$.

Tout cela n'a rien de très neuf et nous ramène, en partie du moins, au jeune Marx, celui des années 1843-1844. Ainsi écrivait-il, dans la Critique de la philosophie du droit de Hegel :

Il faut rendre l'oppression réelle plus dure encore en y ajoutant la conscience de l'oppression, et rendre la honte encore plus honteuse en la livrant à la publicité. Il faut représenter chaque sphère de la société allemande comme la partie la plus honteuse de la société allemande.

Ou dans la conclusion de sa lettre à Ruge, en septembre 1843 :

Il s'agit de se confesser, rien de plus. Pour se faire remettre ses péchés, l'humanité n'a besoin que de les appeler enfin par leur nom.

Pourtant, selon Marx, la critique ne saurait s'épuiser à jouer les Cassandre. En effet, une fois la réalité de l'oppression et de l'exploitation décrite, une fois la conscience, mythifiée, tirée de son sommeil en partie coupable, il s'agit d'extraire de cette réalité son potentiel utopique, déjà là, et de rendre ainsi possible un nouveau rapport au monde :

On verra alors que, depuis longtemps, le monde possédait le rêve d'une chose dont il suffirait de prendre conscience pour le posséder réellement [...]. La critique a saccagé les fleurs imaginaires qui ornent la chaîne, non pour que l'homme porte une chaîne sans rêve ni consolation, mais pour qu'il cueille la fleur vivante ${ }^{8}$.

Cette image du rêve, du "rêve d'une chose », de la «fleur vivante » qu'il s'agirait de cueillir, m'a toujours intrigué, notamment parce qu'elle semble contraster, ou du moins entrer en tension, avec la

7. Telle est, chacun l'aura reconnue, la rhétorique de la mauvaise (et fausse) conscience.

8. Dans Critique de la philosophie du droit de Hegel. On trouve là une formule qui n'est pas sans évoquer celle de l'anarchiste Élisée Reclus [1880] pour qui l'idéal n'est autre que la «fleur de la réalité » dont il s'agit de cueillir le fruit. 
rhétorique de la dénonciation. En effet, à trop vouloir rendre le monde inacceptable, n'en vient-on pas à saccager aussi la fleur vivante, du moins à être incapable de la cueillir ? Et n'est-ce pas d'ailleurs, pour partie, ce que Marx et Engels dénoncent dans ce qu'ils nomment, dans La Sainte Famille, la « Critique critique» ? Pour celle-ci, écrivent-ils, « tout ce qui est réel, tout ce qui est vivant (i.e. tout ce qui relève de l'expérience sensible, plus généralement de toute expérience réelle) est non critique et massif, par conséquent n'est "rien", et seules les créatures idéales, fantasmagoriques de la Critique critique sont "tout" ». Or, pour Marx, la tâche de la critique - la plus radicale -, celle qui prend les choses à la racine, doit au contraire rompre avec de telles spéculations abstraites et doctrinaires, cesser de penser l'idéal comme une sorte d'implant transcendantal, venu d'on ne sait où. Elle doit au contraire révéler en quoi ce qui est contient déjà, à titre de virtualité, ce qui doit être ; donc concevoir l'idéal comme la tendance et le mouvement d'une chose existante.

Ces formules peuvent paraître bien obscures. Mais elles ne sont pas si mystérieuses. Elles consistent à affirmer que la seule objectivité qui se conçoive est celle qui intègre les potentialités des actions, des relations, des situations. Il en résulte une conception nécessairement ouverte, mais aussi généreuse, du monde social. Dans une telle perspective, les idéaux existent bel et bien dans le monde ou la nature, du moins pour celui ou celle qui sait voir. Ce qui suppose que le monde (ou la nature) puisse être hospitalier à nos aspirations. Pour le formuler dans les termes de John Dewey, la critique ainsi doit manifester, sur le modèle de la « complicité originelle » raynalienne [Raynal, 2016, p. 74], une certaine piété face à ce qui est :

En dépit de toutes ses imperfections et de ses défauts, la nature - dans laquelle il faut inclure l'humanité - peut susciter un sentiment de piété en tant qu'elle est la source d'idéaux, de potentialités et d'aspirations et le foyer possible de toutes les formes de bien et d'excellence accessibles [Dewey, 1960, p. 244].

Ainsi, d'une façon paradoxale, critiquer ce qui est suppose d'en donner la meilleure description : celle qui fait droit aux possibilités idéales données dans le monde, et nous offre ainsi les moyens propres à les actualiser pour en cueillir les fleurs vivantes. Au même titre que la littérature ou l'art en général, la sociologie peut être alors conçue, pour reprendre une autre formulation de Dewey, comme « un commentaire sur la nature et la vie en vue 
d'une appréhension plus intense et plus juste des significations accompagnant l'expérience » [Dewey, 2012, p. 368 ${ }^{9}$.

\section{La sociophilie de Cooley : la société comme œuvre d'art et trésor des possibles}

Cette critique de la sociophobie sociologique pourrait sembler bien peu sociologique. N'est-ce pas de science, sociale, qu'il s'agit, tout le reste n'étant que littérature ? Rien n'est moins certain. Sauf à laisser dans l'ombre le premier des grands sociologues américains, Charles Horton Cooley, ce grand amateur de littérature (Shakespeare ou Goethe étaient pour lui parmi les plus grands sociologues), formé à l'école du transcendantalisme d'Emerson - ce philosophe du sublime dans l'ordinaire -, et pionnier avec son ami Dewey du pragmatisme. L'un de ses derniers textes, Social Science, mérite en effet d'être lu comme une profession de foi résolument sociophilique :

En vérité, la science est pour partie une forme d'art. Et ce que nos recherches peuvent apporter de plus précieux, c'est cette capacité à observer le mouvement de la vie humaine dans un esprit généreux, à le recomposer de façon à en saisir l'unité idéale et la beauté [Cooley, 1918, p. 404].

Dans son Journal, à l'automne 1903, il précisait :

Le sociologue doit être tout à la fois un collecteur désintéressé de faits et animé d'une vive sympathie avec le mouvement même de la vie : deux choses difficiles à concilier. Il doit avoir l'état d'esprit d'un naturaliste tout en partageant beaucoup de ces sentiments propres au moraliste et au poète.

9. Dans le chapitre V de cet ouvrage, Dewey commente d'ailleurs un mot d'un poète essayiste anglais du XIX ${ }^{\mathrm{e}}$ siècle, Matthew Arnold, pour qui la poésie est « une critique de la vie». Et il en est ainsi, selon le philosophe, parce que tout art permet, par l'imagination, l'expérience de possibilités qui contrastent avec les conditions réelles. C'est un point qu'il développera dans L'Art comme expérience [2005, p. 396] : «Un sens des possibilités qui ne sont pas réalisées, mais qui pourraient l'être, et qui, lorsqu'elles sont mises en contraste avec les conditions réelles, est la "critique" la plus pénétrante [je souligne] qui puisse être faite de ces dernières. C'est par un sens des possibilités s'offrant à nous que nous prenons conscience des constrictions qui nous enserrent et des poids qui nous oppressent. » C'est notamment par ce biais, poursuit Dewey, que l'art contribue à « refaçonner l'expérience de la communauté dans le sens d'un ordre et d'une unité plus grands ». 
Cette exigence d'une vive sympathie avec le mouvement même de la vie, cet appel à un esprit généreux propre à saisir - à embrasser - ce mouvement comme un tout doté de qualités esthétique et morale, mais aussi ce plaidoyer pour un point de vue qui mêle le naturaliste et le poète ne peut qu'évoquer l'œuvre d'Henri Raynal. Comme si Cooley nous invitait à appréhender le tout social et son mouvement sur le modèle du « merveilleux objectif » de la totalité cosmique raynalienne.

Pour Cooley, en effet, l'humanité, la société et l'ensemble des groupes sociaux qui les composent constituent des totalités organiques, c'est-à-dire des ensembles organisés composés d'éléments interdépendants et animés par la contribution singulière de chacun à la vie du tout. Ainsi, si ces totalités sociales peuvent susciter l'émerveillement, révéler une beauté qui leur est propre, c'est avant tout parce que, à travers les multiples coopérations qui en constituent la texture, elles manifestent une unité différenciée, à l'image de l'harmonie d'un orchestre composé de tout un ensemble d'instruments et de musiciens qui se répondent pour interpréter une œuvre commune. C'est en ce sens qu'il suggère :

Au sens littéral, toute société est d'abord une œuvre d'art, lentement et souvent inconsciemment produite - comme le sont les grandes œuvres -, marquée par des traits certes parfois grotesques et étranges mais d'une beauté inépuisable, source de fascination [Cooley, 1963 (1909), p. 21].

Pour autant, cette fascination n'est pas d'ordre seulement esthétique. En effet, pour Cooley, la sociologie ne vaudrait pas une heure de peine si elle ne nous permettait pas d'appréhender le trésor des possibles à l'œuvre dans les relations interhumaines et les totalités sociales qui les embrassent ${ }^{10}$. Dans ce but, il invite ses lecteurs à percevoir notamment combien leur vie commune la plus ordinaire - celle menée dans ce qu'il nomme les groupes primaires (dans les relations familiales, amicales, de voisinage, bref d'interreconnaissance, qu'Alain Caillé réunit sous le concept de socialité primaire) est la source de tout un ensemble d'idéaux, qu'il qualifie

10. Dévoiler de tels trésors est par ailleurs indissociable de l'objectif explicite qu'il se donne, si proche de celui d'Henri Raynal, de soigner le « pessimisme » de son époque, malade, selon lui, de l'individualisme, de l'utilitarisme et d'une conception désenchantée (et désenchantante) de la science. Voir Henri Raynal, dans Cosmophilie, «Refus de l'enténèbrement » [2016, p. 77-86]. 
d' « idéaux primaires ». Les groupes primaires constituent ainsi la pouponnière (nursery) de la nature humaine [ibid., p. 24], et cela en un double sens. Non seulement c'est en leur sein que s'opère le processus conjoint de socialisation et d'individuation - nous ne saurions être des « îles sans océan » [Raynal, 2016, p. 41], sans l'océan de ces relations qui nous font -, mais, également, c'est par l'expérience familière de ces groupes que se développent selon lui tous les idéaux moraux. Ces deux aspects sont indissociables. Mettre en œuvre ces «idéaux primaires » dans nos relations avec autrui, c'est pour Cooley se réaliser soi-même, s'ouvrir à une vie plus profonde et plus riche.

C'est dans cet esprit, résolument sociophilique, qu'il faut lire son plaidoyer pour la démocratie. Il suggère en effet que ses principes constitutifs de liberté, d'égalité et de solidarité constituent moins des droits naturels abstraits, découverts par quelques philosophes brillants, que des sentiments concrets dont chaque individu fait l'expérience concrète au sein de ces groupes :

D'où tenons-nous nos notions d'amour, de liberté, de justice, etc., que nous appliquons aux institutions sociales ? De philosophies abstraites? Non. Bien davantage, à l'évidence, de la vie effective que nous menons dans ces formes de société élémentaires et largement répandues, dans la famille et les groupes de jeux. [...] Égalité des chances, équité, dévouement et allégeance de tous au bien commun, libre discussion, bienveillance à l'égard des plus faibles : les aspirations à une démocratie idéale - ce qui inclut le socialisme et bien d'autres doctrines - jaillissent naturellement du terrain de jeu et de la communauté de voisinage [Cooley, 1963 (1909), p. 32 et 51].

En ce sens, l'idéal démocratique est déjà là, dans les flancs de la société, au cœur de nos expériences les plus ordinaires, en attente de pouvoir - sur le modèle de la parturition - respirer à l'air libre en s'émancipant des groupes restreints où il reste confiné, pour s'ouvrir à la société dans son ensemble ${ }^{11}$. Dans cette perspective, la sociologie, en vertu même de son ambition critique, doit donc cesser, à la différence d'une certaine sociologie critique, de « calomnier l'expérience ordinaire » pour à l'inverse manifester un « respect pour l'expérience humaine et ses potentialités » [Dewey, 2012,

11. « Une démocratie véritable n'est autre que l'application à grande échelle des principes que nous considérons universellement juste de mettre en œuvre dans les petits groupes » [ibid., p.119]. 
p. 67]. Et ainsi témoigner que ce trésor des possibles - la fameuse « fleur vivante » de Marx que j'évoquais précédemment - est en quelque sorte à portée de main.

\section{"L'instant fugitif où la société prend " : l'épiphanie du social selon Mauss}

Rendre justice à ce qui est, comme je l'ai rappelé dans les termes d'Henri Raynal, c'est aussi, d'un point de vue sociophilique, témoigner de l'inventivité énigmatique à l'œuvre dans la texture même du social, en nous rendant sensibles, par un travail de description méticuleux, à ces petits miracles quotidiens par lesquels se nouent les liens sociaux, à ces purs moments de société où le social apparaît en pleine lumière.

Dans son essai fameux, l'Essai sur le don, Marcel Mauss, tel un peintre ou un photographe, proposait de saisir les grandes fêtes du don des sociétés traditionnelles - potlatch, kula, pilou-pilou sous cette belle formule : «L'instant fugitif où la société prend. » Comme si, dans ces instants, à travers les présents donnés, reçus et rendus, la société manifestait « son aspect vivant»; comme si s'offrait au regard des participants - mais aussi de l'anthropologue qui en est le témoin - le «mouvement du tout », instants fugaces où, ajoutait-il, « les hommes prennent conscience sentimentale d'eux-mêmes et de leur situation vis-à-vis d'autrui» [Mauss, 1989, p. 275]. Il s'agit à l'évidence d'un moment privilégié où le lien social - l'énigme même du questionnement sociologique - se noue in situ, in statu nascendi, dans ce que je nommerai une épiphanie du social - une sociophanie ${ }^{12}$.

12. Je serai tenté de dire qu'être ainsi le témoin - direct ou indirect - de telles épiphanies, d'avoir la chance de saisir de tels instants où la société prend, où le corps social est réalisé, définit le bonheur du sociologue ou de l'anthropologue, la condition de félicité de sa vocation. Y a-t-il en effet un plus beau cadeau, pour un sociologue ou un anthropologue, que de se voir ainsi offrir par son terrain, pour ainsi dire sur un plateau, un pur moment de société ? Cette félicité n'est-elle pas comparable à celle ressentie par un astrophysicien découvrant une planète ou une galaxie jusqu'ici inconnue, pour un botaniste ou un zoologue une espèce rare ou prétendument disparue ? N'appelle-t-elle pas, en retour, sur le modèle raynalien de l' «obligation du témoin » et de l' « apostolat pur », à en donner la meilleure description ? Voir Henri Raynal, notamment [2012, chapitre XII ; et 2016, Livre I, chap. II]. 
Dans son Esquisse d'une théorie de la magie, Mauss évoque une expérience comparable, en décrivant en ces termes les danses d'une tribu maritime de Nouvelle-Guinée :

Ce mouvement rythmique, uniforme et continu, est l'expression immédiate d'un état mental où la conscience de chacun est accaparée par un seul sentiment, une seule idée, hallucinante, celle du but commun. Tous les corps ont le même branle, tous les visages ont le même masque, toutes les voix ont le même cri. [...] À voir sur toutes les figures l'image de son désir, à entendre dans toutes les bouches la preuve de sa certitude, chacun se sent emporté, sans résistance possible, dans la conviction de tous. Confondus dans le transport de leur danse, dans la fièvre de leur agitation, ils ne forment plus qu'un seul corps et qu'une seule âme. C'est alors seulement que le corps social est véritablement réalisé [ibid., p. 126].

Il pourrait m'être objecté que ces rites qui célèbrent ainsi notre vie commune ne sont qu'une affaire de sauvages ; que ces moments d'effervescence collective où la société prend relèvent d'une capacité d'émerveillement et d'une naïveté propres à des sociétés prémodernes, restées prisonnières de ce que Max Weber nommait le jardin enchanté de la magie, cette forêt de symboles où, par analogies et correspondances, tout fait sens. Or, à l'évidence, ne partageons-nous pas aussi de tels moments d'enchantement, ce bonheur banal que nous prenons à la socialité elle-même, pour elle-même, à la manifestation du social comme tel ? Ce bonheur de se lier, tel qu'il s'atteste, par exemple, dans une simple conversation, le sociologue allemand Simmel [1981, p. 157 et 131] invitait à le saisir comme un bonheur de pure forme, où les contenus engagés dans la relation importent moins que ce « jeu de société » où chacun donne et reçoit de l'autre rien d'autre que le « maximum de valeurs sociables (en joie, aide et vivacité) », et où « la matière du discours n'est que le support indispensable des attraits que l'échange vivant de la parole déploie comme tel ». On pourrait multiplier à l'envi les exemples de ces moments concrets, si précieux, où nous faisons l'expérience de tels instants fugitifs où la société prend. 


\section{Au commencement était l'émerveillement : du parti du lumineux au pari du don}

Il est temps désormais de nous diriger vers quelques éléments de conclusion afin de préciser vers quelle anthropologie générale nous conduit cette incursion, en compagnie d'Henri Raynal, du côté lumineux de la force du social. Partons de cet extrait d'Expérience et Nature de John Dewey :

L'une des caractéristiques les plus frappantes de l'expérience humaine en général $[\ldots]$ réside dans l'intérêt qu'elle porte à la fête et aux festivités, à l'ornement, à la danse, au chant, à la pantomime dramatique, aux histoires que l'on raconte [2012, p. 100].

Ce qu'il veut ainsi marquer, c'est combien cette expérience ne saurait être dictée par les seules pressions de la nécessité ou de l'utilité. À l'inverse, elle manifeste la primauté d'activités multiples qui sont à elles-mêmes leur propre fin, qui produisent un plaisir, une joie immédiats - en ce sens esthétiques -, mais qui ne sont éprouvés qu'en collectivité, en vertu de la force du social et de son côté lumineux. C'est la raison pour laquelle Dewey condamne la conception si terne du bonheur des utilitaristes, « de la manière dont ils ont travaillé, filé, tissé, sans jamais voir un seul homme vêtu de joie comme le lys dans les champs »encore une histoire de fleur vivante! Ainsi, poursuit-il, « on voit bien que pour eux le bonheur n'a jamais été qu'une affaire de calcul et d'effort, d'industrie commandée par une comptabilité arithmétique » [ibid.].

À cet économisme comptable s'oppose cet impératif antiutilitariste de gratuité, si caractéristique des sociétés traditionnelles où, souligne Dewey, le corps est paré ${ }^{13}$ avant d'être vêtu, où les maisons sont encore des taudis alors que les temples et les palais rivalisent de beauté. Bref, où le luxe l'emporte sur la nécessité. De la pêche et de la chasse les hommes font un jeu ; tout travail utile est, dans la mesure du possible, métamorphosé par les rites et les cérémonies qui l'accompagnent : «Il est subordonné à l'art », insiste Dewey. Il suggère même que la genèse du langage est à chercher du côté poétique de la vie :

13. Pensons ici à la notion raynaliene d'apparure. Voir notamment «L'apparure (ou Faire un poème de soi) » [Raynal, 2016, p. 151-158]. 
La source de la parole ne réside pas dans le sombre esprit de sérieux, mais dans la griserie du jeu et l'hilarité juvénile. Qui nierait, poursuit-il, que c'est à la littérature plus qu'au commerce et à la science que l'on doit le développement de nos ressources linguistiques? [ibid.,p. 105 ${ }^{14}$ ].

Mettre ainsi l'accent sur le côté poétique de la vie incite à poser une hypothèse assez singulière pour les sciences sociales, mais à laquelle nous invite la cosmophilie raynalienne : et si, au commencement, était l'enchantement, l'émerveillement ? Dewey, grand lecteur de la littérature anthropologique de son temps, suggère que si, dans ces sociétés, le surnaturel domine, c'est parce que la fantasmagorie du surnaturel manifeste la beauté de la pensée, de la forme et du mouvement, abondant en exemples enchanteurs de cohérence logique entre les choses et les êtres, le visible et l'invisible. On retrouve là un thème cher à Henri Raynal : c'est la cohérence de l'univers qui est au principe de sa beauté et de l'enchantement qu'il suscite. Et peu importe ici qu'elle soit perçue par la pensée magique ou mythique ou par les sciences les plus modernes ${ }^{15}$.

Poursuivons plus loin cette hypothèse périlleuse. Cette forêt de symboles, cette théâtralité des rituels des sociétés traditionnelles par lesquelles elles se célèbrent elles-mêmes ne marquent-elles pas combien les pensées et actions humaines ont, d'abord, valeur d'éloge ? Cet éloge, adressé à ce que Durkheim nomme le « divin social », ne manifeste-t-il pas notre gratitude à l'égard de la totalité sociale qui nous embrasse ? L'efficacité des rites, la force du symbolisme ne sauraient donc être recherchées, en premier lieu du moins, dans une quelconque utilité. Elles manifestent avant tout ce par quoi la société se rend présente - se donne - à elle-même, d'une façon immédiate et sensible, pour renforcer ainsi l'unité morale du groupe, le «sentiment du Nous »-We-feeling, dirait

14. Rousseau, dans le premier discours, n'affirmait-il pas déjà : «On nous fait du langage des premiers hommes des langues de géomètres, et nous voyons que ce furent des langues de poètes. [...] Voilà pourquoi les premières langues furent chantantes et passionnées avant d'être simples et méthodiques » [1990, p. 66-67].

15. Plus encore, ces «fantasmagories » ne sont pas sans effets pratiques. Songeons ici au grand texte de Lévi-Strauss consacré à l'« efficacité symbolique ». Ne montret-il pas combien le rôle du shaman vis-à-vis de la parturiente est avant tout d'assigner une forme et un sens là où se déployait auparavant un chaos de sensations brutes et absurdes ? N'est-ce pas la mise en ordre qu'il opère, en attribuant à ce désordre une signification admise par la communauté et la femme enceinte, qui restitue cette dernière à l'ordre humanisé de la nature ? 
Cooley. Comme si l'être du social, dans la profusion des formes par lesquelles il se manifeste, devait se « redoubler » en un paraître ; comme si, pour paraphraser Jacques Dewitte [2010], était inhérent à la vie sociale un pur besoin de se montrer, de s'exhiber, de se donner à voir, de déployer des dispositifs ornementaux, rituels, festifs, ludiques qui, bien loin d'être utiles, peuvent aller à l'encontre de tout principe d'utilité ${ }^{16}$.

Éclairer de la sorte le côté lumineux de la force du social conduit ainsi à proposer un fondement résolument non utilitaire à notre vie commune. Ou plus généralement, et plus radicalement encore, à suggérer que la vie interhumaine repose sur le congédiement de la nécessité (de l'utilité, de la fonctionnalité) ou, pour reprendre une formule d'Henri Raynal [2016, p. 327-328], dans quelques lignes consacrées au sourire, sur une suspension des causalités. Pourquoi ? Parce que la nécessité, c'est ce qui nous divise, nourrit la concurrence vitale ${ }^{17}$. À l'inverse, l'émerveillement, c'est ce qui nous unit, sur le modèle de l'accord raynalien du soi avec l'univers - du moins si, à l'instar de Dewey, on désigne par ce terme la totalité des conditions avec laquelle le soi est en relation. Face aux flots de la nécessité et aux caprices de l'utilité, n'est-ce pas la beauté gratuite, « sans pourquoi », de la forme - tant des formes symboliques que des formes sociales -, qui vient répondre à ce besoin premier de mise en sens de l'existence individuelle et sociale ?

Formulons les choses autrement. Mettre ainsi l'accent sur le côté poétique (ou symbolique) de la vie sociale, c'est souligner combien l'expérience de la coprésence d'autrui, de l'être ensemble suscite d'elle-même l'expérience collective de l'émerveillement et l'ensemble des actes qu'elle invente : « complimenter, louer, chanter, honorer, célébrer, parer, orner, faire œuvre de beauté », comme l'écrit Henri Raynal [2016, p. 222]. Comme si la force généreuse du social - par la puissance nouvelle qu'elle donne à chacun en l'invitant à « sortir de soi » (Mauss) pour s'enrichir, s'élargir, par la confrontation à l'altérité, par sa capacité à produire des idéaux collectifs - appelait un don en retour ${ }^{18}$, qu'il soit adressé

16. Ou être à la source des activités utiles et fonctionnelles. Voir Hocart [2005].

17. Rousseau encore : "L'effet naturel des premiers besoins fut d'écarter les hommes et non de les rapprocher » [1990].

18. À l'instar notamment du poète qui, face à la beauté que l'univers lui prodigue si généreusement, se trouve, sur le modèle du don agonistique maussien, dans l'obligation 
aux puissances invisibles symbolisant le groupe, aux ancêtres et aux morts ou à nos contemporains, qu'il prenne la forme de grandes fêtes rituelles sacrées ou celle des petites cérémonies toutes profanes propres à nos formes de sociabilité quotidiennes modernes.

Pour conclure, je voudrais souligner que rendre ainsi justice au côté lumineux de la force du social n'exige pas de laisser dans l'ombre sa face obscure. Au contraire, un tel regard invite à faire l'hypothèse, fondamentale dans le paradigme du don, que ce qui nous lie - le sym-bolique - contient, au double sens du terme, ce qui nous divise - le dia-bolique ${ }^{19}$. Ou, pour faire image, la lumière - cette dimension morale, épiphanique et esthétique du social - n'apparaît que sur fond d'ombre, celui de la violence. Prendre le parti du lumineux n'occulte donc pas la possibilité, toujours présente, parfois omniprésente, de basculer du côté obscur de la force du social. Simplement, et ce n'est pas rien, ce parti - qui n'est autre que le pari du don - nous invite à penser combien le monde social ne saurait s'instituer et se pérenniser sans cette « générosité, anonyme ou humaine », dont le sociologue, comme le poète, serait bien inspiré de témoigner « de façon à ce qu'elle puisse se montrer en sa légitime évidence » [Raynal, 2010, p. 53].

\section{Références bibliographiques}

\section{Euvres d'Henri Raynal}

- 2016, Cosmophilie. Nouvelles locales du Tout, Cécile Defaut, Lormont.

- 2012, Ils ont décidé que l'univers ne les concernait pas, Klincksieck, Paris.

- 2010, L'Accord, Fata Morgana, Paris.

- 2002, «Entretien avec Henri Raynal », in Missives, Société littéraire de la Poste, Paris.

- 1965, L'Orgueil anonyme, Seuil, Paris.

- 1963, L'Eil magique, Seuil, Paris.

de lui « rendre » ses bienfaits par son œuvre. Voir notamment «L'émulation originelle » et « L'apostolat pur », in Cosmophilie.

19. Voir notamment Caillé [2014] et Chanial [2016]. 


\section{Autres textes}

Boltanski Luc, 2008, Rendre la réalité inacceptable, Demopolis, Paris.

Bourdieu Pierre et al., 1993, La Misère du monde, Seuil, Paris.

CAILlé Alain, 2014, Anti-utilitarisme et paradigme du don. Pour quoi ?, Le Bord de l'eau, Lormont.

Chanial Philippe, 2016, « Du symbolique au diabolique. Ambivalences et normativité du don », in CARrÉ Louis, Loute Alain (dir.), Donner, Reconnaître, Dominer. Trois modèles en philosophie sociale, Presses universitaires du Septentrion, Lille.

- 2011, La Sociologie comme philosophie politique. Et réciproquement, La Découverte, Paris.

Cooley Charles, 1918, Social Process, C. Scriber's \& Son, New York.

- 1963 (1909), Social Organization, Shocken, New York.

Dewey John, 2012 (1925), Expérience et Nature, Gallimard, Paris.

- 2005 (1931), L'Art comme expérience, Farrago, Pau.

- 1960 (1929), The Quest for Certainty, Capricorn, New York.

Dewitte Jacques, 2010, La Manifestation de soi, La Découverte/MAUSS, Paris.

Hocart Arthur M., 2005, Au commencement était le rite, La Découverte/ MAUSS, Paris.

MARx Karl, 1982, Euvres, vol. III, Gallimard, «Bibliothèque de la Pléiade », Paris.

Mauss Marcel, 1989, Sociologie et Anthropologie, PUF, Paris.

Reclus Élisée, 1880 [2007], Évolution et Révolution, rééd. Le Passager clandestin, Lyon.

Renault Emmanuel, 2004, L'Expérience de l'injustice, La Découverte, Paris.

Rousseau Jean-Jacques, 1990, Essai sur l'origine des langues, Gallimard, « Folio essais », Paris.

Simmel Georg, 1981, Sociologie et Épistémologie, PUF, Paris. 